\title{
Analysis of a Hydraulic Pipe System with Major and Minor Pressure Losses
}

\author{
Mohammad Alesmaeel, Ali Alfarsi, Safaa Almusa, Emilio Diaz, Souren Grigoryan, Joao Queda, Tadeh Zirakian \\ and David Boyajian
}

Department of Civil Engineering and Construction Management, California State University, Northridge, CA 91330, USA

\begin{abstract}
Bernoulli's principle states that an increase in the speed of a fluid is directly related to the decrease in the fluid's potential energy. Many engineers refer to Bernoulli's equations to calculate the pressure of a system. The objective of this undergraduate research endeavor is to illustrate the accuracy of his theory and apply it to one of the most common fluid systems in residential homes, a pump pipe system. The research team consisted of a diverse body of undergraduate students with different educational and cultural backgrounds. Completing this objective further improved every member's problem solving, communication skills, self-confidence, ability to rationalize and transcribe physical phenomena as well visually express them to rest of the engineering community. The findings of this research showed a relationship between various parameters such as, pipe length, pipe roughness, diameter, and specific gravity of the liquid.
\end{abstract}

Key words: Bernoulli's equation, pipe flow, hydraulics, educational model, engineering creativity.

\section{Introduction}

An engineer is only as good as their ability to transcribe data through experimentation and come up with an accurate relationship of the parameters in question. This ability can only be developed through repeated exposure to diverse challenges where one must use fundamental engineering techniques and logic to express real world problems in mathematical models. After a model is accurately represented, basic arithmetic should suffice in extracting the information needed. This ability to visualize the model of the problem is what makes experience in the field of engineering so desired. The goal of this endeavor is to further heighten the creativity and understanding of conceptualization to engineering undergraduate students. When Civil Engineers are exposed to any project where fluids are concerned on of the most important parameters is pressure. The criticality for engineers to understand and calculate the pressure

Corresponding author: Tadeh Zirakian, Ph.D., P.E., assistant professor, research fields: civil engineering, engineering education. values of a hydraulic system cannot be overstated. With the help of Bernoulli's equation, a theoretical model of two different points in a control volume can be related through pressure and velocity [1]. To accurately create relationships between various fluid pressure parameter, tables and charts of previous engineering lab tests were collected, including pipe roughness and pipe angle coefficients [2]. This research study explored real life problems by comparing theoretical and experimental values obtained using a physical model, as well as the use of a firsthand experimentation of Bernoulli's concepts in his Hydrodynamica. The objective of this research is to better explore the relationship between initial pressure and its loss within the system, the two types of loss (minor and major), and the volumetric flow rate throughout the whole system [3]. Pressure loss in a hydraulic pipe system can be further classified into minor and major losses with each being a function of different variables. Minor loss is a function of the deviations from a straight pipe flow, meaning all the turns the pipe makes using various connections. While major loss is a function of the dimensions of a certain 
pipe along with the velocity of the liquid passing through it.

\section{Theories and Concepts}

Antoine Laviosar first stated the law of conservation of mass, which states that for any system closed to all transfers of matter and energy, the mass of the system must remain constant over time. As a system's mass cannot change, so quantity cannot be added nor removed [4]. His famous work was later used to derive a multitude of equations including Eq. (1), the continuity equation, which holds true for steady state isentropic flows such as the one evaluated.

$$
Q_{1}=Q_{2}=\rho_{1} V_{1} A_{1}=\rho_{2} V_{2} A_{2}
$$

In Eq. (1), $Q=$ volumetric flowrate $\left(\mathrm{ft}^{3} / \mathrm{s}\right), \rho=$ density of the fluid (slug/ $\left.\mathrm{ft}^{3}\right), V=$ average velocity of fluid $(\mathrm{ft} / \mathrm{s})$, and $A=$ cross sectional area $\left(\mathrm{ft}^{2}\right)$. Bernoulli's equation creates a simplistic model by grouping certain properties together, to create different heads of pressure that are all converted into units of height as seen in Eq. (2) [5].

$$
z_{1}+\frac{P_{1}}{\rho g}+\frac{V_{1}^{2}}{2 g}+h_{p}=z_{2}+\frac{P_{2}}{\rho g}+\frac{V_{2}^{2}}{2 g}+h_{L}
$$

In Eq. (2), $Z=$ height of the fluid relative to a constant reference point $(\mathrm{ft}), P=$ pressure of fluid $\left(\mathrm{lbf} / \mathrm{ft}^{2}\right), g=$ acceleration due to gravity $\left(\mathrm{ft} / \mathrm{s}^{2}\right), h_{p}=$ pressure head of pump (ft), and $h_{L}=$ the total pressure head loss (ft). The various pressure heads were identified as gravity, static, velocity, and pump respectively. Each pressure head contributes to the total possible pressure in the system, which according to Bernoulli, should be equal on both sides of an incompressible and isentropic flow (adiabatic and reversible process). His initial equation has expanded to both pumps and friction losses in a closed system. The mathematical representation of both major pressure loss and minor pressure loss can be seen in Eq. (3) and Eq. (4), respectively.

$$
\begin{aligned}
& h_{L-\text { major }}=f \frac{(L)\left(V^{2}\right)}{2(g)(D)} \\
& h_{L-\text { minor }}=\Sigma K_{f} \frac{V^{2}}{2 g}
\end{aligned}
$$

In Eqs. (3) and (4), $f=$ Darcy's friction factor, $L=$ length (ft), $V=$ average velocity of fluid (ft/s), $g=$ acceleration due to gravity $\left(\mathrm{ft} / \mathrm{s}^{2}\right), D=$ diameter of the pipe (ft), $\Sigma K_{f}=$ loss coefficient for pipe components. With all the variables known for minor loss, the Darcy's friction factor was the key missing element to complete the pressure loss equations. Darcy's friction factor is a factor that is dependent on what type of flow is present in the system as there are three different types of flow which behave differently. Laminar flow which is generally seen when dealing with: small pipes, relatively low velocities, is characterized when the innermost parts flow the fastest and the cylinder touching the pipe is not moving at all. Turbulent flow however is generally found in high flow rate systems with large pipes and is unpredictable due to its high velocity. In between both type of flows there is the transitional flow which is a mixture of laminar and turbulent flow. With turbulence in the center of the pipe, and laminar near the edges, each of these flows behaves in different manners in terms of frictional energy loss while flowing and has different equations that predict their behavior. To differentiate between the flows Reynolds number, a unitless parameter is used to identify the flow's behavior. This is calculated using Eq. (5).

$$
R e=\frac{\rho V L}{\mu}
$$

where $\rho=$ density of the fluid (slug/ $\left./ \mathrm{ft}^{3}\right), V=$ average velocity of fluid (ft/s), $L=$ length (ft), and $\mu=$ dynamic viscosity of the fluid (lbf.s/ $/ \mathrm{ft}^{2}$ ).

With Reynolds number known, the appropriate equation can be applied to calculate $f$, Darcy's friction factor, which can be later used to calculate the major pressure loss. A flow is considered laminar if the Reynolds number is below 2,000, turbulent flow is the term given to flows with a Reynolds number greater than 4,000, and transitional flow is for flows in between laminar and turbulent. The correct equation can be evaluated by using Eqs. (6)-(8).

$$
\text { Laminar Flow: } f=\frac{64}{R e}
$$


Transitional Flow: $f=\frac{1.63}{\ln ^{2}\left[\frac{6.9}{R e}+\left(\frac{\epsilon}{3.7 D}\right)^{1.11}\right]}$

Turbulent Flow: $\frac{1}{\sqrt{f}}=-1.8 \log \left[\frac{6.9}{R e}+\left(\frac{\frac{\epsilon}{D}}{3.7}\right)^{1.11}\right]$

In Eqs. (7) and (8), Re = Reynolds number, $D=$ diameter of the pipe (ft), and $\epsilon=$ relative roughness of pipe. With the correct equation now used and the appropriate friction factor known, Eq. (3) can be used to sum up both pressure losses, and the accumulation to the total loss in the system, $h_{L}$, which is expressed in Eq. (5). With the sum of the losses calculated using Eq. (9), and Bernoulli's equation now complete for five different pump pressures, various relationships between each type of pressure losses, volumetric flow rates, and velocity can be made to make sense of the flow.

$$
h_{L}=h_{L-\text { minor }}+h_{L-\text { major }}
$$

By applying the law of conservation of energy to a fluid system, the exit velocity of the flow was able to be calculated using Eq. (1), which assumes that if the area of the pipe and the density of the liquid are constant, then the velocity of the inlet and outlet should also remain constant if friction pressure loss were to be ignored. With the velocity known for the same system with both a presence of loss (experimental) and an absence of loss (theoretical), the loss from the system due to pressure losses was calculable. Minor loss consists of the pressure difference due to entrances, exits, and different types of connection that deviate the direction of the flow from its customary direction. Where major losses sum up the pressure difference due to the friction coefficient of the material of the pipe, while also considering the dimension of the pipe, and velocity of the flow [6]. However, to calculate the major loss in a system an experimental friction factor, $f$, is needed which is a function of the Reynolds number of the flow. The Reynolds number, pipe coefficient and diameter of the pipe govern the friction factor and equation used. This study deals with a relatively low pipe roughness and low velocity, which translates to a friction coefficient of 0.008 due to its laminar flow which can be found on the Moody Chart. This research provides a simple yet practical approach to understand how a hydraulic system works, which helps engineers design for required pump pressure and efficiency of any hydraulic system.

\section{Details of Physical Model}

To construct the physical model representation of the team's hydraulic system some specific materials were required. The system required five $10^{\prime} 1 / 2$ " diameter PVC pipes, five $90^{\circ}-1 / 2$ " and three $45^{\circ}-1 / 2$ " PVC elbows for connections. A Kedsum $550 \mathrm{GPH}$ electric submersible pump was used to pressurize the system. Two 5-gallon buckets with volumetric lines to record the volume of water going in and out of the system and all-purpose cement glue to assist with neutralization of any pressure losses to mitigate error in the experiment.

To create a system with significant minor and major pressure loss, a large system of pipes and connections had to be constructed, which was computer designed using both AutoCAD and SOLIDWORKS programs. The 5-gallon bucket was first filled above the halfway mark to have enough water for the system. The various pipes were connected to resemble the drawing on Fig. 1. While an overall plan view of the designed system is shown on Fig. 2, and the actual built model is shown in Fig. 3. To secure the piping system, an adhesive was also applied on the inner and outer layers of the connection, to make sure there is no pressure loss that is not accounted for. The pump was placed in the 5-gallon bucket and held down using the legs found on the bottom of the pump, while the other empty bucket was placed at the outlet of the system to record the volume of water coming out of the system. The pump was then turned on to pressurize the system, while also being used to check for any leaks from the pipes and connections prior to the experiment. While one tactician turned the valve open another timed 15 seconds of flow and the valve was then closed. After 


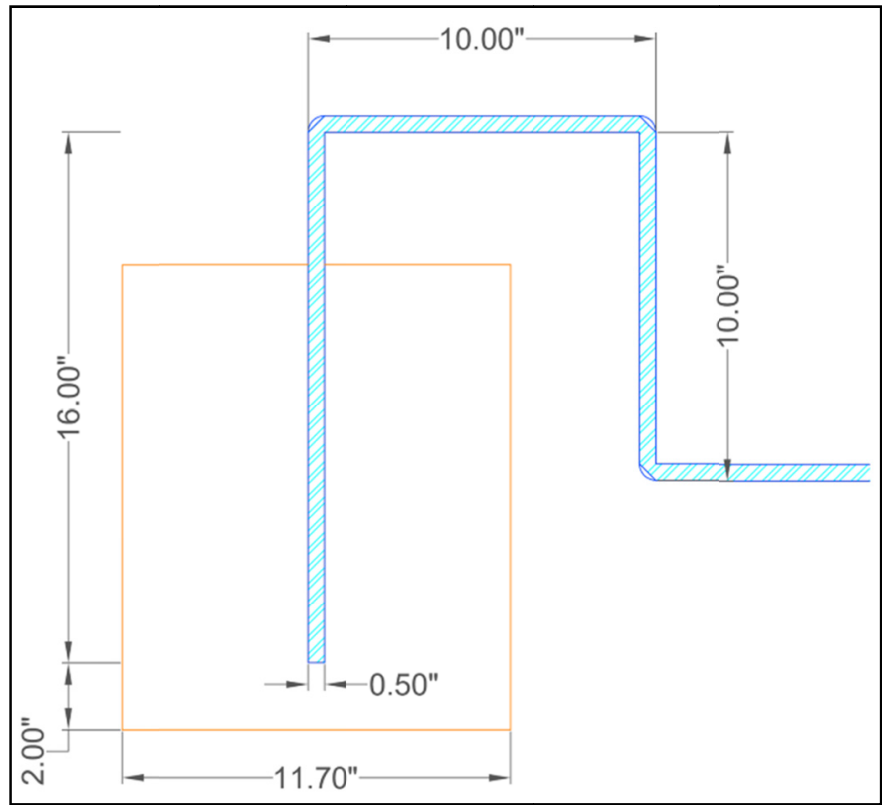

Fig. 1 Elevation view of inlet hydraulic system.

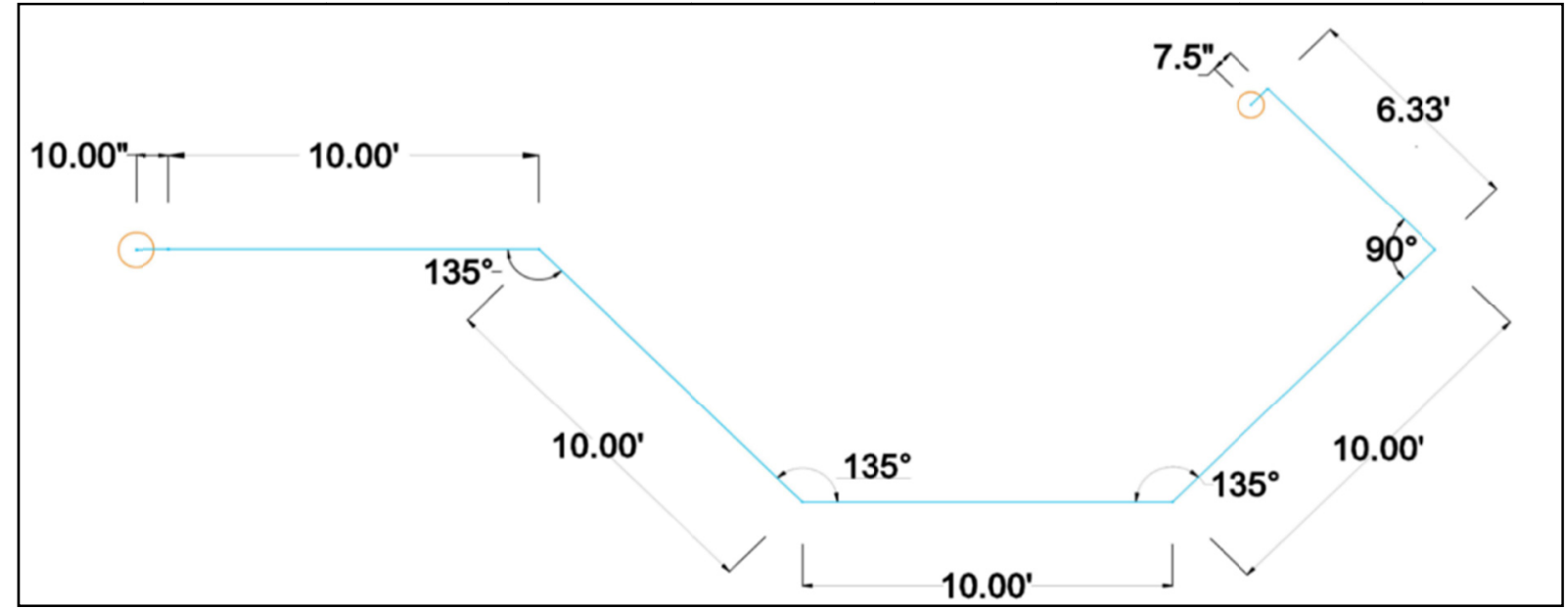

Fig. 2 Plan view of hydraulic system.

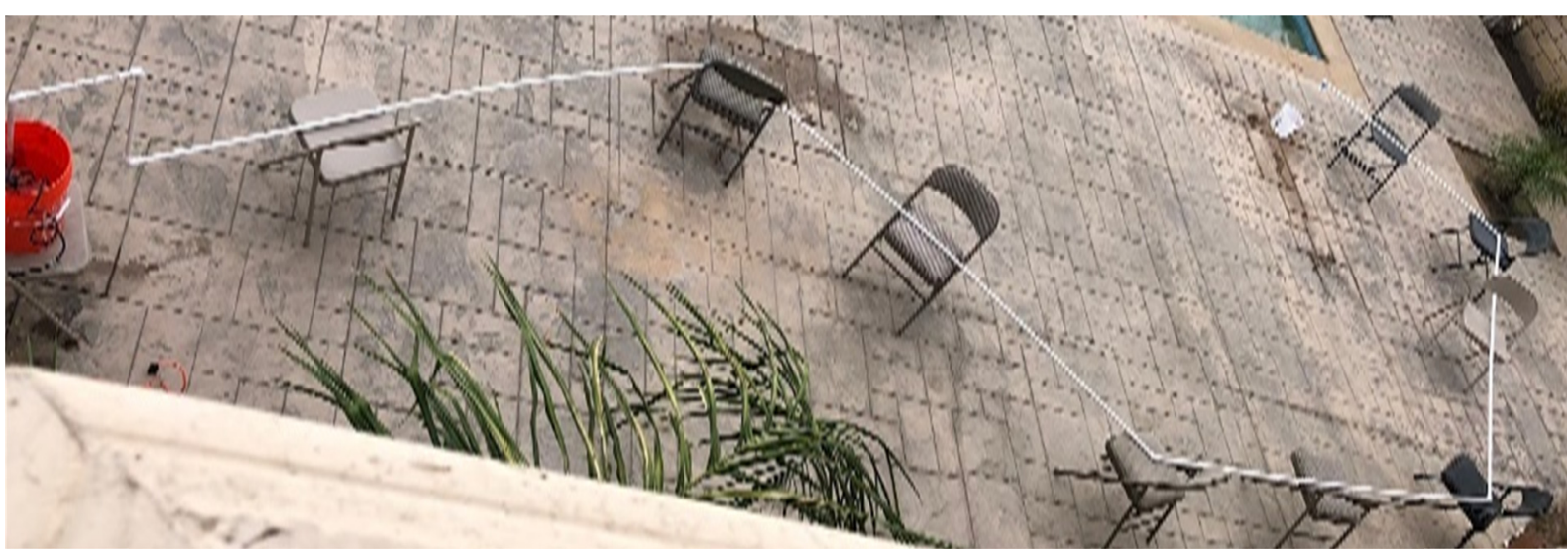

Fig. 3 PVC pipe system used in the research study. 
three trials of each of the five different pressures of 1 , $1.5,2,2.5$, and 3 heads of pressure $\left(h_{p}\right)$, the volumetric flow rate was measured by dividing the volume by the time for each trial. The three trial values for each pressure were averaged out to have consistent volumetric flow rate.

To have a basis of comparison, a system was also constructed with a 4 " PVC pipe following the same steps listed above, but because of its short length the loss due to friction would become so small that it would become negligible. The velocity was then measured using Eq. (1). With both the experimental and theoretical velocities known various calculations and relationships were made using a combination of the equations listed above.

\section{Discussion of Results}

The theoretical predictions and test results are discussed in this section. Table 1 and Table 2 show the relationship between the average velocity through the system and the pressure losses that are followed by it. Minor loss is due to the various connections of the pipe and major loss is due to the length of the pipe and the roughness of the material of the pipe. However, both types of losses are functions of velocity as seen in Eq. (3) and Eq. (4) and are exponentially related to one another. One can see in the pressure loss equations that velocity is squared, therefore the faster the flow the more pressure one can expect to lose to both deviations from a straight path (minor loss) and the friction that the flow experiences from the inside of the pipe containing it (major loss). However, at such low velocities there is still a clear increase in volumetric flow rate $Q$ at the outlet of the system as seen in Table 1 . To further conceptualize the amount of pressure loss that the system experienced it is necessary to look at the percentage variations from the tests ran with only a 4" long pipe and compare the differences. Table 2 depicts the difference in volumetric flow rate that the longer pipe which is exposed to more friction has in relation to the very short pipe where friction is negligible. As velocity of the flow increases so does the volumetric flow rates for both systems but the short pipe with no friction increases at a much steeper rate. At $P=3$ ', the system has a $22.8 \%$ loss of pressure when compared to a no loss system, and at $P=1$ ' there was only a $11.75 \%$ loss due to the friction of the system. This increase in velocity attributes to more loss due to the friction of the surface area of the pipe itself which contacts the water. This can be limited by using a straighter pipe, a larger diameter, less velocity, shorter pipe, and a pipe constructed out of material with a lower friction coefficient. The most important parameter in this is the speed of the fluid since it is the only parameter that is squared in the loss equations. In this experiment the pipe material, length, temperature

Table 1 Velocity vs. pressure losses.

\begin{tabular}{lll}
\hline Velocity $\left(\mathrm{ft} / \mathrm{s}^{2}\right)$ & $H_{L}$ major $(\mathrm{ft})$ & $H_{L} \min (\mathrm{ft})$ \\
\hline 1.102 & 0.184 & 0.0453 \\
1.166 & 0.206 & 0.0507 \\
1.237 & 0.231 & 0.0570 \\
1.314 & 0.261 & 0.0644 \\
1.366 & 0.283 & 0.0677 \\
\hline
\end{tabular}

Table 2 Pressure head vs. $Q$ actual vs. \% loss.

\begin{tabular}{llll}
\hline$H_{p}(\mathrm{ft})$ & $Q_{\text {avg }}$ short pipe $\left(\mathrm{ft}^{3} / \mathrm{s}\right)$ & $Q_{\text {avg }}$ long pipe $\left(\mathrm{ft}^{3} / \mathrm{s}\right)$ & Pressure loss $(\%)$ \\
\hline 1 & 0.00306 & 0.00271 & 11.75 \\
1.5 & 0.00317 & 0.00276 & 13.0 \\
2 & 0.00326 & 0.00281 & 14.4 \\
2.5 & 0.00346 & 0.00287 & 17.0 \\
3 & 0.00377 & 0.00291 & 22.8 \\
\hline
\end{tabular}


and diameter of the pipe were left constant with velocity being the variable by having different pump pressures to isolate one factor for precision.

Figs. 4 and 5 help to visualize how much of an effect the properties of a hydraulic system can have on the output volumetric flow rate. While the total pressure loss due to friction of this hydraulic system is shown in Fig. 6, the goal of a hydraulic system is to transport fluid as efficiently as possible but with the losses in this system show engineering judgment dictates the degree of efficiency that can be obtained.

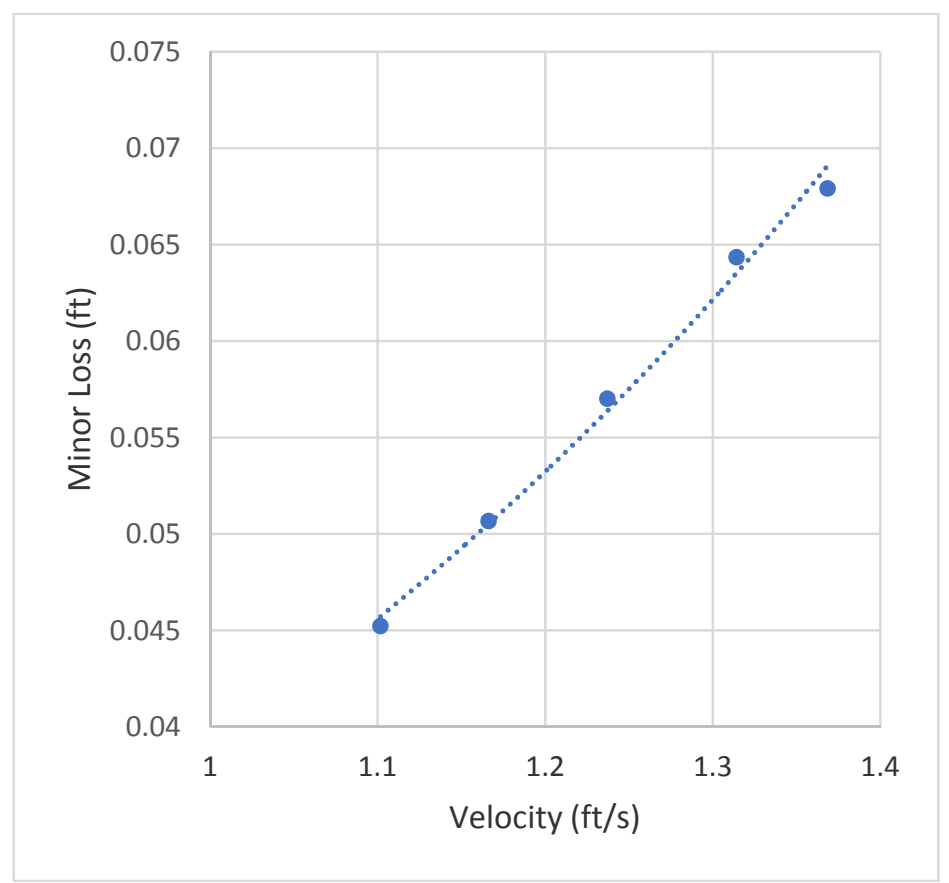

Fig. 4 Minor pressure loss plotted against average velocity.

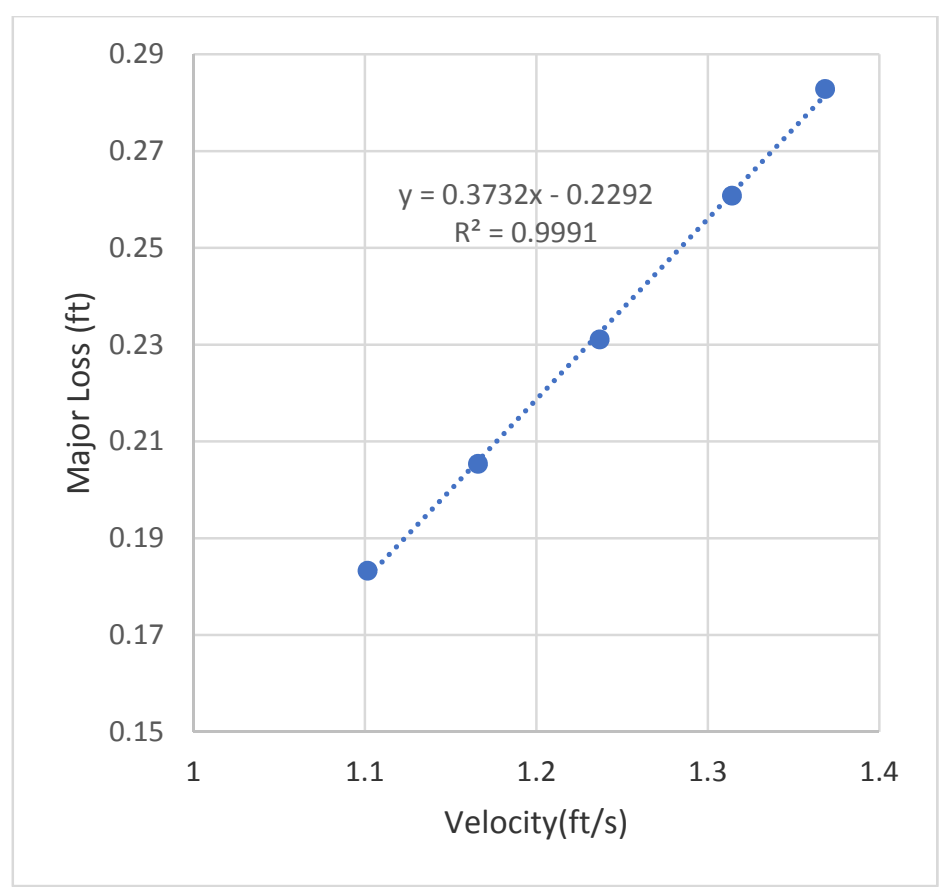

Fig. 5 Major pressure loss plotted against average velocity. 


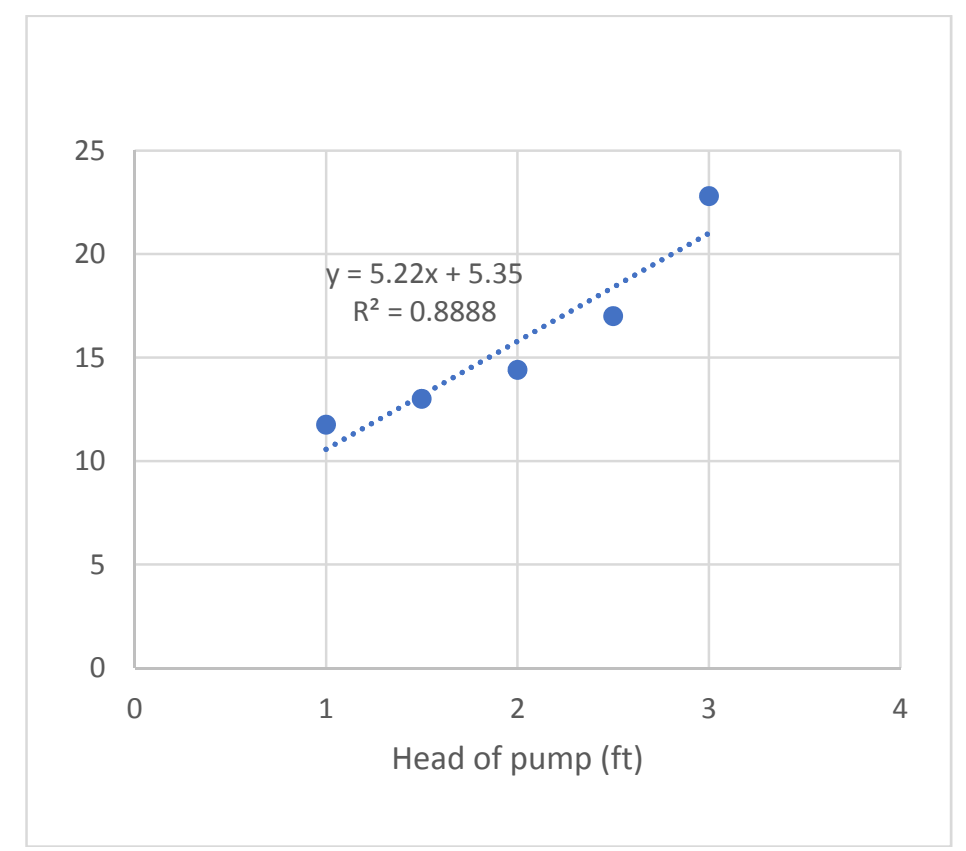

Fig. 6 Total pressure loss due to friction relative to pump pressure.

\section{Educational Objectives}

The primary objective of this research was to better explore and prove the important relationship between, pipe length, pipe roughness, diameter and specific gravity of the liquid, as well explain and classify the minor and major losses of a hydraulic pipe system. The research team is proud to state that in the matter of personal educational achievements a lot of positive outcomes were reached.

This research assignment also provided a view of communication in the field. Working on this project showed how different cultural and ideological backgrounds can positively affect group ideas. There were several different ways to think which were brought into discussions and helped the group in selecting the best pathways to work. Also, by applying the learnings exposed in class sessions, group meetings and the guidance provided by instructors, the members of the group were able to bring the best performance in this research. The skills learned in this project, will be essential in the future careers of the members.

\section{Concluding Remarks}

The experiment sheds some light on pipe flow and its parameters when pressure loss was further examined. The team expressed relationships between parameters such as pipe length, pipe roughness, diameter, specific gravity of the liquid, etc. and how each parameter affects the loss in a system. This is important for engineers to understand to be able to make educated design decisions when considering pressure loss. The pressure losses found in this study are magnified when considering larger systems, with lengths of hundreds or thousands of miles. Even the smallest fraction of a percent of pressure loss is important when designing billion-dollar pipe systems. This experiment helps engineers ask important questions such as, how the material of this pipe will affect its volumetric flow rate at the end of the pipe. Or what is the optimum velocity of flow where the minimum loss occurs while still discharging enough fluid. The answers to these questions are not as simple as this experiment but it is a good basis to apply to real life scenarios in the future.

The comprehension of such topics and one's ability to make correct calculations and decisions accordingly are what separates an average person from an engineer. The reason engineers are respected and compensated as much as they are is because of their ability to apply past 
knowledge from previous experiments into the field and get appropriate results. To be successful in the field of engineering, the arsenal of a well-rounded engineer should consist of understanding of theories presented in the classroom and capability to apply that knowledge into solving real life problems.

\section{Acknowledgments}

The student research team would like to show recognition to Dr. Tadeh Zirakian and Dr. David Boyajian, Professors of Civil Engineering in the Department of Civil Engineering and Construction Management at California State University, Northridge for sharing their knowledge on water recourses and guiding them to successfully complete this research endeavor.

\section{References}

[1] Saleta, M. E., Tobia, D., and Gil, S. 2005. "Experimental Study of Bernoulli's Equation with Losses." American Journal of Physics 73 (7): 598-602. doi: 10.1119/1.1858486.

[2] Lindeburg, M. R. 2018. PE Civil Reference Manual. Kaplan: Professional Publications, Inc.

[3] Çengel, Y. A., and Cimbala, J. M. 2018. Fluid Mechanics: Fundamentals and Applications. New York: McGraw-Hill.

[4] Duveen, D. I., and Klickstein, H. S. 1954. "Medallic Portraiture of Antoine Laurent Lavoisier." Journal of Chemical Education 31 (6): 308-9.

[5] Lindsay, G. A. 1952. "Pressure Energy and Bernoulli's Principle." American Journal of Physics 20 (2): 86-88. doi:10.1119/1.1933123.

[6] Wisniak, J. 2011. "Conservation of Energy Readings on the Origins of the First Law of Thermodynamics. Part II." Educacion Quimica 19 (3): 216. doi: 10.22201/fq.18708404e.2008.3.258. 


\section{Appendix}

The following is a sample calculation to find the effective pressure loss in the system when the head of the pump was set to 3 feet. By applying the givens and lugging into the volumetric flow rate equation the team was able to find the velocity in the system. Finding the velocity is the key to the calculating the Reynolds number and the major and minor pressure losses in this hydraulic system.

$$
\begin{aligned}
& P_{1}=3 f t \\
& Q_{1}=0.00588 \mathrm{ft}^{3} / \mathrm{s} \\
& \rho=1.94 \text { slugs } / \mathrm{ft}^{3} \\
& D=.0416 \mathrm{ft} \\
& \mu=2.34 \mathrm{lbf} * s / f t^{2} \\
& g=32.2 \mathrm{ft} / \mathrm{s}^{2} \\
& L=50.567 \mathrm{ft} \\
& Q=\rho V A \quad \rightarrow V=\frac{Q_{1}}{\rho A}=\frac{00588}{1.94 \times(.00136)}=2.23 \mathrm{ft} / \mathrm{s} \\
& R e=\frac{\mathrm{g} V D}{\mu}=\frac{1.94 \times(2.23) \times(.0416)}{2.34 \times 10^{-5}}=7691.02 \\
& f=0.008 \quad b / c \in \approx 0 \\
& h_{L \min }=\sum K_{L} \frac{V^{2}}{2 g} \\
& h_{L \text { min }}=2.4 \times \frac{(2.23)^{2}}{64.4}=0.185 \\
& h_{L \text { major }}=f \times \frac{L}{D} \times \frac{V^{2}}{2 g} \\
& h_{L \text { major }}=0.008 \times\left(\frac{50.567}{0.0416}\right) \times\left(\frac{2.23^{2}}{64.4}\right)=0.7509 \\
& h_{L}=h_{L \text { min }}+h_{L \text { major }} \\
& h_{L}=0.185+0.7509 \\
& h_{L}=0.9359
\end{aligned}
$$

